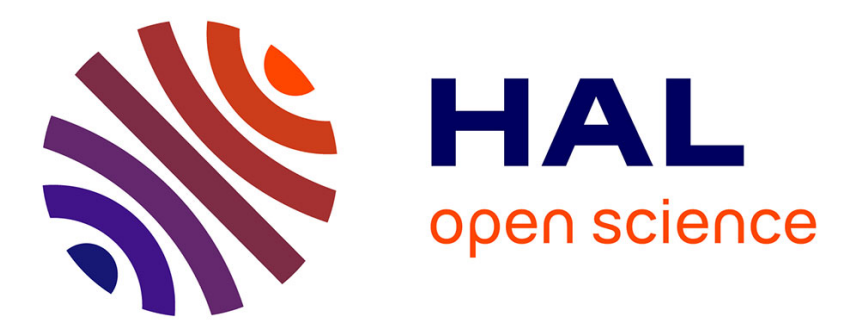

\title{
Improvements in computational aspects of Interval Kalman Filtering enhanced by constraint propagation
}

\author{
Jun Xiong, Carine Jauberthie, Louise Travé-Massuyès
}

\section{To cite this version:}

Jun Xiong, Carine Jauberthie, Louise Travé-Massuyès. Improvements in computational aspects of Interval Kalman Filtering enhanced by constraint propagation. IEEE International Workshop Electronics, Control, Measurement, Signals and Their Application to Mechatronics, Jun 2013, Toulouse, France. 6p. hal-01966341

\section{HAL Id: hal-01966341 \\ https://hal.laas.fr/hal-01966341}

Submitted on 28 Dec 2018

HAL is a multi-disciplinary open access archive for the deposit and dissemination of scientific research documents, whether they are published or not. The documents may come from teaching and research institutions in France or abroad, or from public or private research centers.
L'archive ouverte pluridisciplinaire HAL, est destinée au dépôt et à la diffusion de documents scientifiques de niveau recherche, publiés ou non, émanant des établissements d'enseignement et de recherche français ou étrangers, des laboratoires publics ou privés. 


\title{
Improvements in computational aspects of Interval Kalman Filtering enhanced by constraint propagation
}

\author{
Jun Xiong ${ }^{1,2}$, Carine Jauberthie ${ }^{1,3}$ and Louise Travé-Massuyès ${ }^{1,4}$ \\ ${ }^{1}$ CNRS, LAAS, 7 avenue du Colonel Roche, F-31400 Toulouse, France \\ ${ }^{2}$ Université de Toulouse, INP, LAAS, F-31400 Toulouse, France \\ ${ }^{3}$ Université de Toulouse, UPS, LAAS, F-31400 Toulouse, France \\ ${ }^{4}$ Université de Toulouse, LAAS, F-31400 Toulouse, France \\ E-mail: jxiong@laas.fr, cjaubert@laas.fr, louise@laas.fr
}

\begin{abstract}
This paper deals with computational aspects of interval kalman filtering of discrete time linear models with bounded uncertainties on parameters and gaussian measurement noise. In this work, we consider an extension of conventional Kalman filtering to interval linear models [1]. As the expressions for deriving the Kalman filter involve matrix inversion which is known to be a difficult problem. One must hence find a way to implement or avoid this tricky algebraic operation within an interval framework. To solve the interval matrix inversion problem and other problems due to interval calculus, we propose an original approach combining the set inversion algorithm SIVIA and constraint satisfaction propagation. Several contractors are proposed to limit overestimation effects propagating within the interval Kalman filter recursive structure. Thus the description of our approach is followed by an application and we compare the proposed approach with interval kalman filtering developped in [1].
\end{abstract}

\section{INTRODUCTION}

Set-membership (SM) methods have been the focus of a growing interest and they have been applied to many tasks ([2], [3], [4]). The litterature on this topic shows interesting progress in the last years. SM estimation can be based on interval analysis that was introduced by [5] and several algorithms have been proposed (for more details, see [6], [7], [4]). Other approaches dedicated to linear models include ellipsoid shaped methods ([8], [9]), parallelotope and zonotope based methods [10]. One of the main advantages of the SM estimation approach is that it provides a guaranteed solution in contrast to stochastic estimation approaches. However, it does not give any precision about the belief degree. On the other hand, the SM approach is often criticized for the overestimation of the results. However, one should note that in a similar way, the stochastic approach may estimate system states with a wide confidence range, which may turn as difficult the interpretation of the results. As a matter of fact, both techniques have specific advantages and they may interact synergically. A stochastic method can provide means for analyzing the properties of an SM estimator, and conversely an SM technique can provide the initial entry to a system without assuming the distribution law in advance. They are hence more complementary than competitive. Most importantly, in an estimation framework, the experimental conditions about noise and disturbances are usually properly modeled through appropriate assumptions about probability distributions. However, other sources of uncertainty are not well-suited to the stochastic approach and are better modeled as bounded uncertainty. This is the case of parameter uncertainties that generally arise from design tolerances and from aging. In such cases, combining stochastic and bounded uncertainties may be an appropriate solution.

Motivated by the above observations, we consider the filtering problem for discrete time linear models with bounded uncertainties on parameters and gaussian measurement noise. In [1], the classical Kalman filter [11] has been extended to interval linear models. We consider this work and propose several operations that improve the filtering. In particular, the approach proposed in [1] does not provide guaranteed results because it avoids especially interval matrix inversion by using operations described below. Our contribution consist in proposing an alternative approach to solve the interval matrix inversion problem without loss of solutions while controlling the inherent pessimism of interval calculus. Several technical operations are proposed to limit the overestimation effects due to interval propagation within the interval Kalman filter recursive structure. In particular the gain of the filter is obtained by a calculus based on the set inversion algorithm SIVIA (Set Inversion via Interval analysis) [12] which is combined with constraint propagation techniques.

This paper is organized as follows. Section II describes the problem formulation and the system which is considered. In Section III, a briefly introduction and some main concepts on interval analysis are proposed. An overview of the revised interval kalman filtering algorithm is presented in Section IV, followed by the developed approach to control overestimation. In Section V an example is given and used to compare different approaches. Conclusions and future works are presented in Section VI.

\section{PROBLEM FORMULATION}

In this paper, we consider the unknown state estimation of interval discrete time linear models with gaussian measurement noise. Classically, linear dynamic models are described by the following form:

$$
\left\{\begin{array}{l}
x_{k+1}=A x_{k}+B u_{k}+w_{k}, \\
y_{k}=C x_{k}+D u_{k}+v_{k}, k=0,1,2, \ldots
\end{array}\right.
$$

where $x_{k} \in \mathbb{R}^{n}, y_{k} \in \mathbb{R}^{m}$ and $u_{k} \in \mathbb{R}^{p}$ denote respectively state, observation and input vectors. The matrices $A, B, C$ and $D$ are constant matrices such that $A \in \mathbb{R}^{n \times n}, B \in \mathbb{R}^{n \times p}, C \in$ $\mathbb{R}^{m \times n}$ and $D \in \mathbb{R}^{m \times p}$. In the case of Kalman models ([11]), 
$\left\{w_{k}\right\}$ and $\left\{v_{k}\right\}$ are independent centered gaussian white noises sequences, with covariance matrices $Q$ and $R$, respectively, definite positive by definition:

$$
\begin{aligned}
& E\left\{w_{k}, w_{l}\right\}=Q \delta_{k l}, E\left\{v_{k}, v_{l}\right\}=R \delta_{k l}, \\
& E\left\{w_{k}, v_{l}\right\}=E\left\{w_{k}, x_{0}\right\}=E\left\{v_{k}, x_{0}\right\}=0, \\
& \forall k, l=0,1,2, \ldots
\end{aligned}
$$

where $\delta_{k l}$ is the Kronecker symbol.

To estimate the unknown states of (1) and based on the idea motivated in the introduction, we propose to combine two aspects of modeling: measurement and system noises are still modeled in a stochastic framework and parameters are modeled as bounded uncertainties. System (1) is then reformulated in this double context where the matrices $A, B, C$ and $D$ are bounded interval matrices. The core problem in this work is the unknown state estimation of (1) rewritten in an interval form.

The concept of interval matrices is explained in the following section. In this section, some classical concepts of interval analysis are reminded. These concepts are mainly taken from [13] and [6].

\section{INTERVAL ANALYSIS}

\section{A. Preamble}

The key idea of interval analysis is to reason about intervals instead of real numbers and boxes i.e. interval vectors instead of real vectors. The first motivation was to obtain guaranteed results from floating point algorithms and it was then extended to validated numerics [5]. Let us recall that in computers, real numbers can only be represented by a floating point approximation, hence introducing a quantification error. A guaranteed result means first that the result set encloses the exact solution. The width of the set, i.e. the result precision, may be chosen depending on various criteria among which response time or computation costs. Secondly, it also means that the algorithm is able to conclude on the existence or not of a solution in limited time or number of iterations.

\section{B. Main concepts}

The problem is to wrap the sets of interest into boxes or union of boxes. There are some fundamental operations on intervals which are briefly explained after the definition of an interval.

1) Interval: A real interval $[p]=[p, \bar{p}]$ is a closed and connected subset of $\mathbb{R}$ where $p$ represents the lower bound of $[p]$ and $\bar{p}$ represents the upper bound. The width of an interval $[p]$ is defined by $w(p)=\bar{p}-\underline{p}$, and its midpoint by $m(p)=$ $(\bar{p}+p) / 2$.

The set of all real intervals of $\mathbb{R}$ is denoted $\mathbb{I}$.

Two intervals $\left[p_{1}\right]$ and $\left[p_{2}\right]$ are equal if and only if $p_{1}=p_{2}$ and $\overline{p_{1}}=\overline{p_{2}}$. Real arithmetic operations are extended to intervals [5].

Arithmetic operations on two intervals $\left[p_{1}\right]$ and $\left[p_{2}\right]$ can be defined by:

$\circ \in\{+,-, *, /\},\left[p_{1}\right] \circ\left[p_{2}\right]=\left\{\alpha \circ \beta \mid \alpha \in\left[p_{1}\right], \beta \in\left[p_{2}\right]\right\}$.

An interval vector (or box) $[X]$ is a vector with interval components and may equivently be seen as a cartesian product of scalar intervals:

$$
[X]=\left[\alpha_{1}\right] \times\left[\alpha_{2}\right] \times \ldots \times\left[\alpha_{n}\right] .
$$

The set of $n$-dimensional real interval vectors is denoted by $\mathbb{I R}^{n}$.

An interval matrix is a matrix with interval components. The set of $n \times m$ real interval matrices is denoted by $\mathbb{I} \mathbb{R}^{n \times m}$. The width $w($.$) of an interval vector (or of an interval matrix) is$ the maximum of the widths of its interval components. The midpoint $m($.$) of an interval vector (resp. an interval matrix)$ is a vector (resp. a matrix) composed of the midpoint of its interval components.

Classical operations for interval vectors (resp. interval matrices) are direct extensions of the same operations for punctual vectors (resp. punctual matrices) [5].

2) Inclusion function: Given $[p]$ a box of $\mathbb{I R}^{n}$ and a function $f$ from $\mathbb{I R}^{n}$ to $\mathbb{I} \mathbb{R}^{m}$, the inclusion function of $f$ aims at getting an interval containing the image of $[p]$ by $f$.

The range of the function $f$ over $[p]$ is given by:

$$
f([p])=\{f(\alpha) \mid \alpha \in[p]\} .
$$

The interval function $[f]$ from $\mathbb{I} \mathbb{R}^{n}$ to $\mathbb{I}^{m}$ is an inclusion function for $f$ if:

$$
\forall[p] \in \mathbb{I}^{n}, f([p]) \subset[f]([p]) .
$$

An inclusion function of $f$ can be obtained by replacing each occurrence of a real variable by its corresponding interval and by replacing each standard function by its interval evaluation. Such a function is called the natural inclusion function. In practice the inclusion function is not unique, it depends on the syntax of $f$.

3) Inclusion test: Given a subset $S$ of $\mathbb{R}^{n}$, we test if $[\alpha]$ belongs to $S$, more precisely if $[\alpha] \subset S$ or $[\alpha] \cap S=\emptyset$. These tests are used to prove that all points in a given box satisfy a given property or to prove that none of them does.

4) Contractor: The last operation is the contraction of $[\alpha]$ with respect to $S$. This means that we search a smaller box $[\gamma]$ such that $[\alpha] \cap S=[\gamma] \cap S$. If $S$ is the feasibility set of a problem and $[\gamma]$ turns out empty, then the box $[\alpha]$ may not contain the solution [12]. These operations are used to test if a box can or cannot be removed from the solution set. When no conclusion can be drawn, the box may be bisected and each of the sub-boxes can be tested in turn (this corresponds to branch-and-bound algorithms).

\section{SIVIA: Set Inversion Via Interval Analysis}

Consider the problem of determining a solution set for the unknown quantities $u$ defined by

$$
\begin{aligned}
S & =\{p \in P \mid \Phi(p) \in[\beta]\}, \\
& =\Phi^{-1}([\beta]) \cap P,
\end{aligned}
$$

where $[\beta]$ is known a priori, $P$ is an a priori search set for $p$ and $\Phi$ a nonlinear function not necessarily invertible in the classical sense. (2) involves computing the reciprocal image of $\Phi$. This can be solved using the algorithm SIVIA, which is a recursive algorithm that explores all the search space without loosing any solution. This algorithm makes it possible to derive a guaranteed enclosure of the solution set $S$ as follows:

$$
\underline{S} \subseteq S \subseteq \bar{S} .
$$

The inner enclosure $S$ is composed of the boxes that have been proved feasible. To prove that a box $[p]$ is feasible it is 
sufficient to prove that $\Phi([p]) \subseteq[$ beta $]$. Reversely, if it can be proved that $\Phi([p]) \cap[\beta]=\emptyset$, then the box $[p]$ is unfeasible. Otherwise, no conclusion can be reached and the box $[p]$ is said undetermined. The latter is then bisected in two subboxes that are tested until their size reaches a user-specified precision threshold $\varepsilon>0$. Such a termination criterion ensures that SIVIA terminates after a finite number of iterations.

However, the number of bisections to be performed is generally prohibitive. Hence, recent algorithms take advantage of constraint propagation techniques to reduce the width of the boxes to be tested by the algorithm SIVIA [14], [15], [16]. In this context, the inclusion relations and the equations can be interpreted as constraints. The solution of such system can then be obtained by formulating a Constraint Satisfaction Problem $(C S P)$ as defined below.

A Constraint Satisfaction Problem $H=(\mathcal{X}, \mathcal{D}, \mathcal{C})$ is defined by:

- $\quad$ a set of variables $\mathcal{X}=\left\{x_{1}, \ldots, x_{n}\right\}$,

- $\quad$ a set of nonempty domains $\mathcal{D}=\left\{D_{1}, \ldots, D_{n}\right\}$ where $D_{i}$ is the domain associated to the variable $x_{i}$,

- a set of constraints $\mathcal{C}=\left\{C_{1}, \ldots, C_{m}\right\}$, so that the associated variables to each constraint are defined in $\mathcal{X}$

For solving a $C S P$, different types of so-called contractors can be used [6], [17]. Among the most well-known is the forward-backward contractor [18] which is based on constraint propagation and consists in contracting the domain of the CSP by taking into account any one of the $\left\{C_{1}, \ldots, C_{m}\right\}$ constraints in isolation.

\section{INTERVAL KALMAN FILTERING}

In this section, we consider models described by the form (1) in which the matrices $A, B, C, D$ are interval matrices noted $[A],[B],[C]$ and $[D]$.

In [1], as in the conventional filter, the Kalman Filter Equations provide a minimum variance estimator over all unbiased estimators. Thus an interval Kalman filtering algorithm is proposed. This algorithm is based on interval conditional expectation for interval linear systems. It has the same structure as the conventional Kalman filter algorithm while preserving the statistical optimality and the recursive computational scheme in the SM context. The drawback of this algorithm comes from the fact that it is suggested to use the upper bound of interval matrices to avoid the singularity problems in interval matrix inversion. This point of view leads to a sub-optimal solution that does not preserve guaranteed results, some solutions being lost. We note sIKF this algorithm (suboptimal interval Kalman filtering). It can be noticed that sIKF does not include recent advances in interval analysis and constraint propagation techniques. This is why we propose a new recursive estimator including these recent advances. For simplicity, we consider that the matrix $D$ in (1) is equal to zero.

\section{A. Conventional Kalman filtering}

There are several ways to deduce Kalman equations [11]. One can use mathematical curve-fitting function of data points from a least-squares approximation [19] or also use probabilist methods such as the Likelihood function to maximize the conditional probability of the state estimate from measurement incomes [20]. We consider in the following:

1) $\hat{x}_{k+1 \mid k} \in \mathbb{R}^{n}$ the a priori state estimate vector at time $k+1$ given state estimate at time $k$,

2) $\hat{x}_{k \mid k} \in \mathbb{R}^{n}$ the a posteriori state estimate vector at time $k$ given observations at time $k$,

3) $P_{k+1 \mid k} \in \mathbb{R}^{n \times n}$ the a priori error covariance matrix,

4) $P_{k \mid k} \in \mathbb{R}^{n \times n}$ the a posteriori error covariance matrix.

$P_{\text {.|. }}$ is a key indicator that defines the estimated accuracy of the state estimate, it is characterized as:

$$
P_{l \mid k}=E\left(\left(x_{l}-\hat{x}_{l \mid k}\right)\left(x_{l}-\hat{x}_{l \mid k}\right)^{T}\right),
$$

with $l=k$ or $k+1$

It is known that the Kalman filtering algorithm contains two steps for each iteration: a prediction phase and a correction phase (see for example in [19]).

\section{B. Interval Kalman filtering}

In the SM context, we remind that the matrices $A, B, C$ and $D$ are considered as interval matrices, noted $[A],[B],[C]$ and $[D]$. Notice that $x_{0 \mid 0}, P_{0 \mid 0}, u_{k}, y_{k}$ could be boxes due to the measurement errors or instrument precision. Since punctual values can be considered as interval values for which width is equal to zero, the system framework can be unified for both interval values and punctual values. In the following, we evaluate the impact of changes between the conventional and the interval Kalman filter.

1) Estimation error covariance: in the SM context, the estimation error covariance matrix is an interval matrix which can be rewritten as:

$$
\left[P_{l \mid k}\right]=E\left(\left(\left[x_{l}\right]-\left[\hat{x}_{l \mid k}\right]\right)\left(\left[x_{l}\right]-\left[\hat{x}_{l \mid k}\right]\right)^{T}\right),
$$

where $l=k$ or $k+1 .\left[P_{k \mid k}\right]$ is the estimation error covariance and $\left[P_{k+1 \mid k}\right]$ is the prediction error covariance. All elements on the diagonal of $P_{\text {.|. }}$ are positive as they represent the variance of each state, thus the trace of $P_{\text {.|. }}$ is positive. In the case of an interval matrix, this constraint must also hold. If interval calculus generates intervals containing non positive values, these are spurious and may be removed. Thus a first constraint is introduced which respect to the variance definition: each component of the diagonal of $\left[P_{. \mid}.\right]$noted $\left[P_{. \mid .}\right]_{(i, i)}$ is positive, $i=1,2 \ldots, n$.

2) Prediction step: After defining error covariance in the SM context, the prediction step is investigated. Calculus for the a priori state estimate vector is inherited directly from the determinate model, while corresponding variables are replaced by boxes:

$$
\left[\hat{x}_{k+1 \mid k}\right]=[A]\left[\hat{x}_{k \mid k}\right]+[B]\left[u_{k}\right] .
$$

The possible a priori state estimate based on the previous state estimate and current system input is obtained by this equation. At the previous time $k$, the estimation error is characterized 
by $\left[P_{k \mid k}\right]$. The prediction model does not include noise so the estimation error should also be updated:

$$
\left[\hat{P}_{k+1 \mid k}\right]=[A]\left[\hat{P}_{k \mid k}\right]\left[A^{T}\right]+Q .
$$

This equation can be interpreted as the obtention of all possible a priori estimation error covariances between real state and $a$ priori state estimate at time $k+1$. But as seen previously, the prediction error covariance should respect the fact that $\left[\hat{P}_{k+1 \mid k}\right]_{(i, i)} \geqslant 0, i=1,2 \ldots, n$, which is not guaranteed in interval arithmetic. A CSP is therefore introduced:

$$
\left\{\begin{array}{l}
{\left[\hat{P}_{k+1 \mid k}\right]=[A]\left[\hat{P}_{k \mid k}\right]\left[A^{T}\right]+Q,} \\
\mathcal{C}:\left[\hat{P}_{k+1 \mid k}\right]_{(i, i)} \geqslant 0, i=1,2 \ldots, n .
\end{array}\right.
$$

3) Correction step: from [1], the correction equation holds in the SM context:

$$
\left[\hat{x}_{k+1 \mid k+1}\right]=\left[\hat{x}_{k+1 \mid k}\right]+K_{k+1}\left(\left[y_{k+1}\right]-\left[\hat{y}_{k+1 \mid k}\right]\right) .
$$

Intuitively, $K_{k+1}$ aims to bring back the estimate enclosure around the real state while still retaining all the possible values corresponding to uncertainty. In (9), $K_{k+1}$ is considered as a symbolic varibale, which is shown to be an interval matrix later. Equations (5) and (9) give the estimation error covariance expression. This manipulation is only valid when $E\left\{v_{k}\right\}=0$ :

$$
\begin{aligned}
{\left[\hat{P}_{k+1 \mid k+1}\right]=} & {\left[\hat{P}_{k+1 \mid k}\right]-K_{k+1}[C]\left[\hat{P}_{k+1 \mid k}\right] } \\
& -\left[\hat{P}_{k+1 \mid k}\right]\left(\left[C^{T}\right] K_{k+1}^{T}\right. \\
& +K_{k+1}\left([C]\left[\hat{P}_{k+1 \mid k}\right]\left(\left[C^{T}\right]+R\right) K_{k+1}^{T} .\right.
\end{aligned}
$$

We find $K_{k+1}$ that minimizes trace $\left(\left[\hat{P}_{k+1 \mid k+1}\right)\right]$. The reason why using $\operatorname{trace}(\cdot)$ is that state variance given by the matrix diagonal elements is in fact the value that indicates the estimation error:

$$
\begin{aligned}
\frac{\partial \operatorname{trace}\left(\left[\hat{P}_{k+1 \mid k+1}\right]\right)}{\partial K_{k+1}} & =-2\left[\hat{P}_{k+1 \mid k}\right]\left[C^{T}\right] \\
& +2 K_{k+1}\left([C]\left[\hat{P}_{k+1 \mid k}\right]\left[C^{T}\right]+R\right) . \\
\frac{\partial^{2} \operatorname{trace}\left(\left[\hat{P}_{k+1 \mid k+1}\right]\right)}{\partial K_{k+1} \partial K_{k+1}^{T}} & =2\left([C]\left[\hat{P}_{k+1 \mid k}\right]\left[C^{T}\right]+R\right) .
\end{aligned}
$$

The second derivative is always positive in the conventional kalman filter, which guarantees the existence of a minimization solution. In the SM context, this condition must be forced by a constraint of the same type as (8).

From the first order derivative, we have:

$$
K_{k+1}=\left[\hat{P}_{k+1 \mid k}\right]\left[C^{T}\right]\left([C]\left[\hat{P}_{k+1 \mid k}\right]\left[C^{T}\right]+R\right)^{-1} .
$$

In this case, $K_{k+1}$ turns out to be an interval matrix, which we can note as $\left[K_{k+1}\right] \in \mathbb{I}^{n \times m}$. Thus equations (10) and (11) give the estimation error covariance expression:

$$
\left[\hat{P}_{k+1 \mid k+1}\right]=\left(I_{n}-\left[K_{k+1}\right][C]\right)\left[\hat{P}_{k+1 \mid k}\right] .
$$

Notice that equation (11) involves a matrix inversion, which means the following should be fulfilled:

$$
0 \notin \operatorname{det}\left([C]\left[\hat{P}_{k+1 \mid k}\right]\left[C^{T}\right]+R\right) .
$$

By using the updated state estimate and estimation error covariance, we can proceed to the next iteration.
4) Algorithm loop: Equations (6), (7), (11), (12) and (9) constitute a discrete interval Kalman filter algorithm.

\section{Initialization:}

$P_{0 \mid 0}=\operatorname{Cov}\left\{x_{0}\right\}, m_{0}=E\left(x_{0}\right)$

$\left[x_{0}\right] \sim \mathrm{N}\left(m_{0}, P_{0 \mid 0}\right)$,

Prediction:

$\left[\hat{x}_{k+1 \mid k}\right]=[A]\left[\hat{x}_{k \mid k}\right]+[B]\left[u_{k}\right]$

$\left[\hat{P}_{k+1 \mid k}\right]=[A]\left[\hat{P}_{k \mid k}\right]\left[A^{T}\right]+Q, \quad k=0,1,2, \ldots$

\section{Correction:}

$\left[K_{k+1}\right]=\left[\hat{P}_{k+1 \mid k}\right]\left[C^{T}\right]\left([C]\left[\hat{P}_{k+1 \mid k}\right]\left[C^{T}\right]+R\right)^{-1}$,

$\left[\hat{P}_{k+1 \mid k+1}\right]=\left(I_{n}-\left[K_{k+1}\right][C]\right)\left[\hat{P}_{k+1 \mid k}\right]$,

$\left[\hat{x}_{k+1 \mid k+1}\right]=\left[\hat{x}_{k+1 \mid k}\right]+K_{k+1}\left(\left[y_{k+1}\right]-\left[\hat{y}_{k+1 \mid k}\right]\right), k=$ $0,1,2, \ldots$

A major issue in the SM context is the pessimism introduced by interval arithmetic. Uncertainty is cumulated at each iteration and the interval matrix inversion is time consuming, sometimes divergent. To reduce this pessimism, the techniques developped in the following subsection are used in our approach.

\section{Over estimation control}

1) Gain value propagation: The interval matrix $\left([C]\left[P_{k+1 \mid k}\right]\left[C^{T}\right]+R\right)$ in equation (11) may have a singularity and inversion algebraic operation is difficult. Besides, the interval matrix inverse is obtained by approximation algorithms, like in [21] and is generally over estimated.

We suggest an approach which uses the algorithm SIVIA. The idea is to solve the interval matrix inversion problem by a list of constraint propagation problems. Equation (11) is rewritten as:

$$
\left[K_{k+1}\right]\left([C]\left[\hat{P}_{k+1 \mid k}\right]\left[C^{T}\right]+R\right)=\left[\hat{P}_{k+1 \mid k}\right]\left[C^{T}\right] .
$$

We define $\left[S_{k+1}\right]=[C]\left[\hat{P}_{k+1 \mid k}\right]\left[C^{T}\right]+R,\left[T_{k+1}\right]=$ $\left[\hat{P}_{k+1 \mid k}\right]\left[C^{T}\right]$ and:

$$
\left[K_{k+1}\right]\left[S_{k+1}\right]=\left[T_{k+1}\right]
$$

where $\left[K_{k+1}\right] \in \mathbb{I}^{n \times m}, \quad\left[S_{k+1}\right] \in \mathbb{I}^{m \times m}, \quad\left[T_{k+1}\right] \in$ $\mathbb{I} \mathbb{R}^{n \times m}$.

Each component in matrix $\left[K_{k+1}\right]$ is considered separately and the search space is the cartesian product of each component:

$$
\left[K_{k+1}\right]_{1,1} \times\left[K_{k+1}\right]_{1,2} \times \ldots \times\left[K_{k+1}\right]_{n, m} .
$$

This search space is bisected and tested under SIVIA properly adapted to matrix operation. The result is a set of small boxes that satisfy Equation (15).

Each box provides a "small acceptable gain", the set of boxes is then injected into the correction step to update the covariance matrix and the state estimate vector. The final result is the hull of all covariance matrices and state estimate vectors corrected by each small gain. 
2) Constraint Propagation: In [17], some traditional algorithms for CSP are described.

In the presented work, we use the forward-backward algorithm. The principle is to decompose the constraint equation $f\left(\left[x_{1}\right], \ldots,\left[x_{n}\right]\right)=0$ in a sequence of elementary operations of primitive functions like $\{+,-, *, /\}$ and obtain a list of primitive constraints ([22]). For example, consider the following equation:

$$
\left[\hat{x}_{k+1 \mid k+1}\right]=\left[\hat{x}_{k+1 \mid k}\right]+\left[K_{k+1}\right]\left(\left[y_{k+1}\right]-[C]\left[\hat{x}_{k+1 \mid k}\right]\right) .
$$

This equation can be decomposed into the set of following primitive constraints:

$$
\left\{\begin{array}{l}
a_{1}=[C]\left[\hat{x}_{k+1 \mid k}\right], \\
a_{2}=\left[y_{k+1}\right]-a_{1}, \\
a_{3}=\left[K_{k+1}\right] a_{2}, \\
{\left[\hat{x}_{k+1 \mid k+1}\right]=\left[\hat{x}_{k+1 \mid k}\right]+a_{3} .}
\end{array}\right.
$$

We want to contract $\left\{\left[\hat{x}_{k+1 \mid k+1}\right],\left[\hat{x}_{k+1 \mid k}\right],\left[K_{k+1}\right]\right\}$ by the propagation of constraints without changing $\left\{[C],\left[y_{k+1}\right]\right\}$ because they are considered as inputs.

3) Interval intersection rule: As the associative law is no longer valid in interval arithmetic, we must redefine the product of three and four interval matrices [23]. This is the principle of the interval intersection rule.

$$
\prod_{i=1}^{n}\left[M_{i}\right] \triangleq\left[\left(\prod_{i=1}^{n-1}\left[M_{i}\right]\right) \cdot\left[M_{n}\right]\right] \cap\left[\left[M_{1}\right] \cdot\left(\prod_{i=1}^{n-1}\left[M_{i+1}\right]\right)\right] .
$$

wher $\left[M_{1}\right], \ldots,\left[M_{n}\right]$ are interval matrices.

4) Adaptative calibration: Again, by the nature of interval arithmetic, the width of the resulting interval always tends to increase. Thus, the state estimate is getting wider and wider. A calibration can be implemented to reset the iteration for limiting divergence [23]. When the interval matrix to be inverted is not regular, the calibration takes place:

$$
\left[\hat{x}_{k}\right] \triangleq \hat{x}_{k}+\left[\zeta_{k}\right],\left[P_{k}\right]=\left[P_{0}\right] .
$$

where $\hat{x}_{k}$ is the conventional Kalman state estimate from the nominal system and $\left[\zeta_{k}\right]$ is set from the state variance

In next section, we implement these techniques on a simple example and we compare with the state estimation obtained by using the original interval Kalman filtering proposed in [1].

\section{NUMERICAL SIMULATION}

In this section, we apply previously presented filters on an example proposed in [1], originally proposed in [24] and given by the following form:

$$
\left\{\begin{array}{l}
x_{k+1}=[A] x_{k}+w_{k}, \\
y_{k}=[C] x_{k}+v_{k}, k=0,1,2, \ldots
\end{array}\right.
$$

where $w_{k}$ and $v_{k}$ are gaussian noises, with zero means and covariance matrices $[Q]$ and $[R]$ given by $[Q]=Q+\triangle Q$ and $[R]=R+\triangle R$. Moreover, we have $[A]=A+\triangle A$ and $[C]=C+\triangle C$ where:

$$
A=\left[\begin{array}{cc}
0.4 & 0.1 \\
-0.1 & 0.2
\end{array}\right], C=\left[\begin{array}{ll}
0 & 1
\end{array}\right], Q=\left[\begin{array}{cc}
10 & 0 \\
0 & 10
\end{array}\right], R=1
$$

The bounded perturbations and initial conditions are:

$$
\begin{aligned}
& \triangle A=\left[\begin{array}{cc}
{[-0.1,0.1]} & {[-0.15,0.15} \\
0 & {[-0.25,0.25]}
\end{array}\right], \triangle C=[0 \quad[-0.1,0.1]] \\
& \triangle Q=\left[\begin{array}{cc}
{[-2,2]} & 0 \\
0 & {[-2,2]}
\end{array}\right], \triangle R=[-0.9,1.1] \\
& E\left\{x_{0}\right\}=\left[\begin{array}{l}
1 \\
1
\end{array}\right], \operatorname{Cov}\left\{x_{0}\right\}=\left[\begin{array}{ll}
0.5 & 0.0 \\
0.0 & 0.5
\end{array}\right] .
\end{aligned}
$$

In the following, we want to compare the results provided by three filters: the original interval Kalman filter (noted IKF), its sub-optimal version (sIKF), and our improved approach (iIKF). Let us introduce the variables $N, O$ and $D$ where $N$ is the number of calibration times, $O$ is the number of times for which the interval state estimate does not contain the real state, and $D$ is the norm describing the distance between interval estimate bounds and the true value. $D$ is obtained by:

$$
\left\{\begin{array}{l}
D=\frac{\sqrt{\sum_{k=1}^{K} d\left(\left[\hat{x}_{k}\right], x_{k}\right)^{T} d\left(\left[\hat{x}_{k}\right], x_{k}\right)}}{\sqrt{\sum_{k=1}^{K} x_{k}^{T} x_{k}}}, \\
d\left(\left[\hat{x}_{k}\right], x_{k}\right)=\left(\overline{\left|\left[\hat{x}_{k}\right]-x_{k}\right|}+\underline{\left|\left[\hat{x}_{k}\right]-x_{k}\right|},\right.
\end{array}\right.
$$

where $K$ represents the maximal iteration number. $D$ and $O$ indicate the efficiency of algorithm. Besides, the algorithm SIVIA requires a user-specified precision threshold $\epsilon$, which is the bisection factor. This adjustable parameter is also analysed. $\epsilon=1$ means that there is no gain value propagation taking place. $t$ is the execution time. By using the toolbox Intlab of Matlab ([25]), the results are given in Table I: The results of

\begin{tabular}{|c|c|c|c|c|c|}
\hline Filter & $\epsilon$ & $\mathbf{N}$ & $\mathbf{O}$ & $\mathbf{D}$ & $\mathbf{t}$ \\
\hline \hline IKF & - & 20 & 14 & 575.38 & $0.83 \mathrm{~s}$ \\
\hline sIKF & - & 0 & 56 & 0.85 & $0.75 \mathrm{~s}$ \\
\hline iIKF & 1 & 0 & 0 & 3.07 & $0.91 \mathrm{~s}$ \\
& 0.2 & 0 & 0 & 2.60 & $46 \mathrm{~s}$ \\
& 0.05 & 0 & 0 & 2.56 & $784 \mathrm{~s}$ \\
\hline
\end{tabular}

RESULTS FOR N, O AND D USING IKF AND IIKF WITH DIFFERENT BISECTION FACTORS $\epsilon$.

Table I are consistent with those shown in Figures 1 and 2. We can see that the original IKF has the largest $D$ while suboptimal IKF has the minimum $D$ value, which is explained by the narrow bounds for the interval estimates. But since it replaces the uncertainty matrix to be inverted by its upper bound, some solutions are lost, which leads to the largest value of $O$ : the real state is outside the estimated interval state half of the time.

By using iIKF, $D$ is larger than with sIKF, because it retains all solutions. We notice that the real state and the optimal estimate provided by the conventional Kalman filter are both always contained in boundaries of the iIKF state estimate. The gain value propagated from SIVIA actually refines the interval estimation value, but it is more time consuming as the predefined precision increases. Compared to the original IKF, the iIKF prevents unnecessary recalibration due to the divergent interval operations; compared to sIKF, iIKF retains all the solutions consistent with the bounded error uncertainty. The iIKF hence represents a good compromise.

\section{CONCLUSIONS}

In this contribution, an efficient approach to solve the interval matrix inversion problem is proposed, taking part 


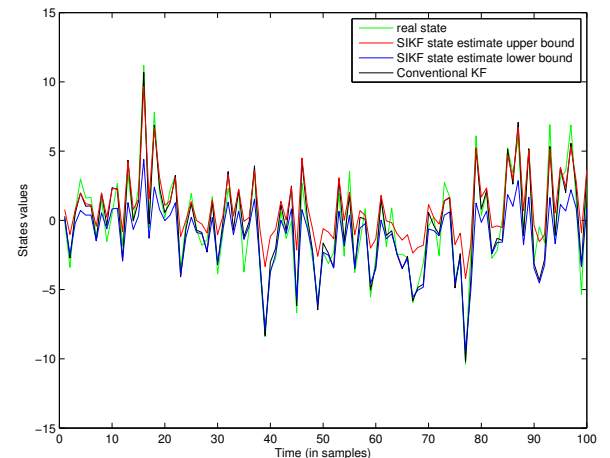

Fig. 1. Simulation results from original sub-optimal IKF

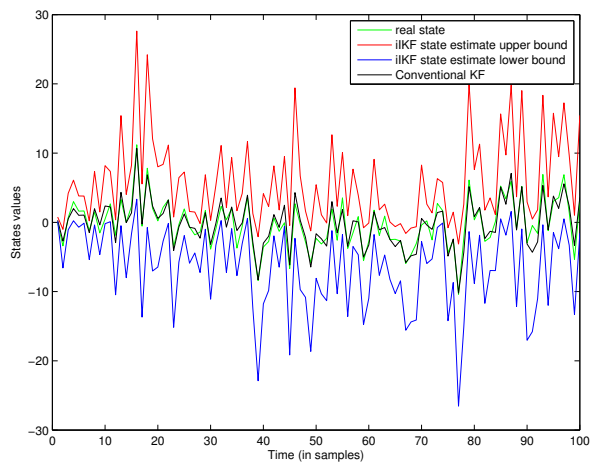

Fig. 2. Simulation results from improved IKF with $\epsilon=0.05$

in an improved version of the interval Kalman filter. This approach leads to matrix inversion without loss of solutions while controlling the inherent pessimism of interval calculus. Several other techniques have also been implemented to limit the overestimation effect due to interval propagation within the interval Kalman filter recursive structure, in particular constraints on the interval covariance matrix have been added. Moreover, the gain of the filter is obtained by a calculus based on the set inversion algorithm SIVIA complemented by constraint propagation. The results have shown that the improved interval Kalman filter (iIKF) includes all the solutions consistent with bounded errors and achieves good overestimation control. Further improvements should target to improve the algorithm efficiency and speed.

\section{REFERENCES}

[1] G. Chen, J. Wang, and L. Shieh, "Interval kalman filtering," IEEE Transactions on Aerospace and Electronic Systems, vol. 33, no. 1, pp. 250-259, Jan. 1997.

[2] E. Gelso, G. Biswas, S. Castillo, and J. Armengol, "A comparison of two methods for fault detection: a statistical decision, and an intervalbased approach," in In proceedings of 19th International Workshop on Principles of Diagnosis DX, Blue Montains, Australia, 2008, pp. 261268.

[3] L. Jaulin, I. Braems, M. Kieffer, and E. Walter, "Nonlinear state estimation using forward-backward propagation of intervals in an algorithm," Scientific Computing, Validated Numerics, Interval Methods, pp. 191204, 2001.
[4] M. Kieffer, L. Jaulin, E. Walter, and D. Meizel, "Guaranteed mobile robot tracking using interval analysis," in In Proceedings of MISC'99, Girona, Spain, 1999, pp. 347-360.

[5] R. E. Moore, Interval analysis. Prentice-Hall, Englewood Cliffs, Jan. 1966.

[6] L. Jaulin, M. Kieffer, O. Didrit, and E. Walter, Applied interval analysis: with examples in parameter and state estimation, robust control and robotics, 1st ed., ser. An emerging paradigm. Springer-Verlag, 2001.

[7] P. Ribot, C. Jauberthie, and L. Trave-Massuyes, "State estimation by interval analysis for a nonlinear differential aerospace model," in In Proceedings of European Control Conference, Kos, Greece, 2007, pp. 4839-4844.

[8] M. Milanese and C. Novara, "Nonlinear Set Membership prediction of river flow," Systems \& Control Letters, vol. 53, no. 1, pp. 31-39, Sept. 2004.

[9] S. Lesecq, A. Barraud, and K. Dinh, "Numerical accurate computations for ellipsoidal state bounding," in In Proceedings of MED'03, Rhodes, Greece, 2003.

[10] A. Ingimundarson, J. Manuel Bravo, V. Puig, T. Alamo, and P. Guerra, "Robust fault detection using zonotope-based set-membership consistency test," International Journal of Adaptive Control And Signal Processing, vol. 23, no. 4, pp. 311-330, 2009.

[11] R. Kalman, "A new approach to linear filtering and prediction problems," Journal of basic Engineering, 1960.

[12] L. Jaulin and E. Walter, "Set inversion via interval analysis for nonlinear bounded-error estimation," Automatica, vol. 29, pp. 1053-1064, 1993.

[13] R. Moore, Automatic error analysis in digital computation. Technical report LMSD-48421, Lockheed Missiles and Space Co, Palo Alto, CA, 1959.

[14] D. Waltz, "Generating semantic descriptions from drawings of scenes with shadows," The Psychology of Computer Vision, pp. 19-91, 1975.

[15] J. Cleary, "Logical arithmetic," Future Computing Systems, vol. 2(2), pp. 125-149, 1987.

[16] E. Davis, "Constraint propagation with interval labels," Artificial Intelligence, vol. 32(3), pp. 281-331, 1987.

[17] G. Chabert and L. Jaulin, "Contractor programming," Artifical Intelligence, vol. 173, pp. 1079-1100, 2009.

[18] F. Benhamou, F. Goualard, F. Granvilliers, and J. Puget, "Revising hull and box consistency," in Proceedings of the International Conference on Logic Programming, Las Cruces, New Mexico, 1999, pp. 230-244.

[19] G. Welch and G. Bishop, "An Introduction to the Kalman Filter," in SIGGRAPH, Los Angeles, California USA, 2001.

[20] C. Masreliez and R. Martin, "Robust bayesian estimation for the linear model and robustifying the Kalman filter," IEEE Transactions on Automatic Control, vol. 22, no. 3, 1977.

[21] J. Rohn, "Inverse interval matrix," SIAM Journal on Numerical Analysis, vol. 30, no. 3, pp. 864-870, June 1993.

[22] O. Lhomme, R. La Chantrerie, A. Gotlieb, and M. Rueher, "Boosting the interval narrowing algorithm," in Proceedings of the 1996 Joint International Conference and Syposium on Logic Programming. Bonn, Germany: The MIT Press, 1996, pp. 378-392.

[23] B. Li, C. Li, and J. Si, "Interval recursive least-squares filtering with applications to video target tracking," Optical Engineering, vol. 47, no. 10, p. 106401, 2008.

[24] X. F. Zhang, A. W. Heemink, and J. C. H. van Eijeren, "Performance robustness analysis of Kalman filter for linear discrete-time systems under plant and noise uncertainty," International journal of systems science, vol. 26, no. 2, pp. 257-275, Feb. 1995.

[25] S. Rump, "INTLAB - INTerval LABoratory," in Developments in Reliable Computing, T. Csendes, Ed. Dordrecht: Kluwer Academic Publishers, 1999, pp. 77-104, http://www.ti3.tu-harburg.de/rump/. 